'Servicio de Cirugía Plástica y Quemados, Hospital Dr. Ernesto Torres Galdames, Iquique, Chile.

IInstituto de Estudios de la Salud, Universidad Arturo Prat. Iquique, Chile. ${ }^{3}$ Centro de Informática Médica y Telemedicina, Facultad de Medicina, Universidad de Chile. Santiago, Chile. ${ }^{4}$ Departamento de Cirugía, Hospital Clínico Universidad de Chile. Santiago, Chile.

${ }^{5}$ Servicio de Imagenología, Hospital Dr. Ernesto Torres Galdames. Iquique, Chile.

${ }^{6}$ Departamento de Oncología Básico Clínico, Facultad de Medicina, Universidad de Chile. Santiago, Chile. ${ }^{7}$ Posta rural Camiña, Chile.

${ }^{8}$ Servicio Anatomía Patológica, Hospital Dr. Ernesto Torres Galdames. Iquique, Chile.

Trabajo no recibió financiamiento.

Los autores declaran que ninguno tiene conflictos de interés.

Recibido el 15 de febrero de 2020, aceptado el 28 de septiembre de 2020.

Correspondencia a: Dr. Marcelo Fonseca C. Chipana 2040, Depto 503. Iquique, Chile. mfon777@hotmail.com

\section{Prevalencia de colecistolitiasis y cáncer de vesícula en una población rural aymara de Chile}

\author{
MARCELO FONSECA C. ${ }^{1,2}$, PABLO BÁEZ B. ${ }^{3}$, ATTILA CSENDES J. ${ }^{4}$, \\ SERGIO CALCAGNO Z. ${ }^{5}$, KATHERINE MARCELAIN C. ${ }^{6}$, \\ PABLO VARGAS M. ${ }^{7}$, JOSÉ GARCÍA R. ${ }^{8}$, TERESA BARLARO F. ${ }^{8}$
}

\section{Prevalence of cholelithiasis and gallbladder cancer among Aymara individuals}

Background: The prevalence of cholelithiasis and gallbladder cancer may be different across ethnic groups. Aim: To study the prevalence of cholelithiasis and gallbladder cancer among Aymara individuals. Material and Methods: An abdominal ultrasound was carried out in a sample of 182 Aymara women aged $46 \pm 16$ years and 76 Aymara men aged $55 \pm$ 16 years. In addition, the histopathological reports of both patients with a history of previous cholecystectomy and those operated after the study were reviewed. Results: Ultrasound was normal in 150 participants (58\%), 76 had cholelithiasis (30\%) and 32 (12\%) had a history of cholecystectomy. Pathological reports of the excised gallbladder were available for 106 cases and showed a chronic cholecystitis in $98 \%$ of cases. Gallbladder cancer was not reported. Conclusions: There is a $42 \%$ prevalence of cholelithiasis and no gallbladder cancer in this sample of Aymara population.

(Rev Med Chile 2020; 148: 1398-1405)

Key words: Cholelithiasis; Epidemiology; Gallbladder Neoplasms; Ethnic Groups. $\tau$ a colecistolitiasis representa un importante problema de salud pública en el mundo y particularmente en Chile, debido a su alta prevalencia, morbimortalidad y a los costos relacionados a la atención de sus variadas formas de complicaciones ${ }^{1-3}$. El primer estudio que demostró la prevalencia de la colecistolitiasis en adultos chilenos se publicó en el año 1971, en un análisis de 3092 autopsias, demostrando que $28 \%$ de los hombres y $60 \%$ de las mujeres presentaban cálculos en la vesícula o habían sido colecistectomizados, con presencia de coledocolitiasis en $10 \%{ }^{4}$. Un año después, otro estudio confirmó los mismos hallazgos ${ }^{5}$. Por otra parte, estudios clínicos y epidemiológicos demuestran que la colecistolitiasis es el principal factor de riesgo para el desarrollo de cáncer de la vesícula biliar6,7, teniendo Chile una de las mayores prevalencias de esta neoplasia en el mundo y representando, actualmente, la tercera causa de muerte por tumores malignos en mujeres chilenas ${ }^{8-10}$. Sin embargo, estas cifras globales no representan la realidad de la población indígena chilena, y solo existen datos referentes a la población mapuche y maorí ${ }^{11}$. No hay referencias sobre la prevalencia de colecistolitiasis y cáncer de la vesícula biliar (CVB) en la población aymara, pueblo originario americano.

Los aymaras son un pueblo andino milenario cuyo territorio se extiende desde las orillas del lago Titicaca, pasando por el altiplano boliviano y la cordillera de la Región de Arica y Parinacota, Tarapacá y terminando en el noroeste de Argentina $^{12,13}$. Las comunas rurales de Colchane $y$ Camiña están conformadas mayoritariamente por 
población aymara en 98 y 78\%, respectivamente. Están ubicadas en la cordillera de los Andes a 4000 metros de altura sobre el nivel del mar en Colchane $y$, a lo largo de la quebrada de Tana, a una altura entre los 2000 y 3200 metros sobre el nivel del mar en Camiña, ambos están aproximadamente a 2000 kilómetros al norte de Santiago de Chile. Presentan altos niveles de pobreza (51\% y $11,6 \%$ respectivamente), baja escolaridad, alto nivel de analfabetismo ( $22 \%$ y $13 \%$, respectivamente) y un acceso deficiente a la atención terciaria de salud $^{14-16}$.

Los objetivos del presente estudio fueron determinar la prevalencia de colecistolitiasis en la población aymara mayor de 18 años, mediante la ecografía abdominal y la prevalencia de CVB en los informes de biopsias de pacientes aymara sometidos a colecistectomía

\section{Material y Método}

\section{Pacientes estudiados}

El presente estudio es de tipo transversal y descriptivo, realizado en una muestra de voluntarios aymaras, mayores de 18 años, pertenecientes a las comunas rurales de Colchane y Camiña, Región de Tarapacá, entre el 01 de abril de 2014 y el 30 de noviembre de 2018. Todos los sujetos dieron su consentimiento informado para realizarse una ecotomografía abdominal. El único criterio de exclusión fue la existencia de una cirugía gastro-esofágica previa, condición que favorece el desarrollo de colecistolitiasis en una alta proporción de casos. Además, se registraron las variables demográficas: nombres y apellidos, género, edad, peso, talla y antecedentes clínicos.

\section{Selección de pacientes}

Para seleccionar y diferenciar la población aymara de la no perteneciente a esta etnia, se definieron 3 criterios: a) tener apellido(s) de origen aymara; b) residencia en territorio tradicional aymara y c) estar acreditado como aymara ante la Corporación Nacional de Desarrollo Indígena (CONADI).

\section{Método}

La ecotomografía abdominal se realizó utilizando un ecógrafo portátil modelo LOGIQ $^{\mathrm{TM}} \mathrm{e}$ (GE Healthcare, General Electric, Chicago. IL, USA) con un transductor 4C. La totalidad de estos exámenes fue realizada por un radiólogo en las respectivas comunidades. Todas las mediciones se efectuaron en una ocasión.

\section{Parámetros evaluados}

Los resultados fueron definidos como: a) hallazgos normales: ausencia de cálculos vesiculares; b) presencia de colecistolitiasis y c) colecistectomía previa. Estas dos últimas condiciones se interpretaron como un resultado positivo para la patología litiásica de la vesícula biliar.

\section{Seguimiento}

Todos los pacientes con diagnóstico de colecistolitiasis fueron derivados al Servicio de Cirugía del Hospital Dr. Ernesto Torres Galdames de Iquique, siendo operados en su totalidad gracias a un proyecto social independiente asociado a esta investigación. Todas las cirugías fueron de tipo electiva. En forma paralela, se revisó el informe histopatológico de los pacientes previamente sometidos a colecistectomía y el informe posterior al diagnóstico de colecistolitiasis y la consiguiente cirugía.

\section{Análisis estadístico}

Todos los datos fueron incorporados en una plantilla Excel y examinados y verificados por dos personas en forma independiente para mayor precisión. Para el análisis de los datos se utilizó el software R-3.4.1. La prevalencia de colecistolitiasis se informó como porcentaje. Las variables en escala de razón se agruparon de acuerdo a cuartiles generales. Se emplearon dos pruebas estadísticas para comparar las distribuciones de los valores de las variables: Chi cuadrado de Pearson para variables de escala nominal y Jonckheere-Terpstra para variables de escala ordinal. Para la significación estadística se definió un $\mathrm{p}<0,05$.

El presente estudio obtuvo la aprobación del Comité Ético-Científico del Servicio de Salud de Iquique

\section{Resultados}

\section{Prevalencia de colecistolitiasis por género}

La muestra se compuso de 258 individuos aymara, asintomáticos, mayores de 18 años; de los cuales $70,5 \%(\mathrm{n}=182)$ eran mujeres y $29,5 \%$ $(\mathrm{n}=76)$ eran hombres. De los 258 pacientes incluidos en este estudio, $150(58,1 \%)$ tuvieron 
una ecografía normal, 76 (29,5\%) presentaron colecistolitiasis y $32(12,4 \%)$ tenían el antecedente de colecistectomía por enfermedad litiásica biliar, en los cuales se comprobó ecográficamente la ausencia de vesícula biliar. Estas dos últimas condiciones, asumidas previamente como positivas para enfermedad litiásica biliar, sumaron 108 pacientes, resultando una prevalencia global de colecistolitiasis de $41,9 \%$, la cual se divide en una prevalencia por género de $48 \%$ en mujeres y $28 \%$ en hombres, valor que no alcanzó significación estadística (Tabla 1).

\section{Distribución por género y edad}

La edad promedio de las mujeres fue de 45,6 $\pm 16,4$ años y de los hombres de $55,1 \pm 15,9$ años
( $\mathrm{p}>0,25)$. En la Tabla 2 se aprecia que no hay diferencia en la distribución de edad según si los sujetos son normales o tienen patología litiásica, tanto en mujeres como hombres. Se presenta la distribución de la muestra por edad por cuartiles generales y dicotómica (menores y mayores de 49 años). No hubo diferencias significativas en la prevalencia de colecistolitiasis comparando los sujetos de ambas localidades (Camiña y Colchane).

\section{Índice de masa corporal}

La distribución de los sujetos con o sin patología biliar según su índice de masa corporal (IMC) demuestra que los individuos con obesidad tenían significativamente mayor prevalencia de colecistolitiasis comparados con sujetos normales (Tabla 3).

Tabla 1. Tasa de prevalencia de cálculos biliares

\begin{tabular}{|c|c|c|c|c|c|c|c|}
\hline \multirow[b]{2}{*}{ Hallazgo } & \multicolumn{2}{|c|}{$\begin{array}{l}\text { Mujeres } \\
(n=182)\end{array}$} & \multicolumn{2}{|c|}{$\begin{array}{c}\text { Hombres } \\
(n=76)\end{array}$} & \multicolumn{2}{|c|}{$\begin{array}{c}\text { Total } \\
(n=258)\end{array}$} & \multirow[t]{2}{*}{$\mathbf{p}^{\S}$} \\
\hline & $\mathbf{n}$ & P (\%) & $\mathbf{n}$ & P (\%) & $n$ & P (\%) & \\
\hline Normal & 95 & 52 & 55 & 72 & 150 & 58,1 & \\
\hline Cálculos biliares & 60 & 33 & 16 & 21 & 76 & 29,5 & \\
\hline Colecistectomía & 27 & 15 & 5 & 7 & 32 & 12,4 & \\
\hline CL total & 87 & 48 & 21 & 28 & 108 & 41,9 & $0,006^{*}$ \\
\hline
\end{tabular}

P: Tasa de prevalencia. Datos ajustados por la edad. CL: Colecistolitiasis. \$Prueba de Chi cuadrado de Pearson. *Diferencias significativas entre mujeres y hombres.

Tabla 2. Distribución de los individuos con y sin colecistolitiasis por género y edad

\section{a. Distribución de la edad por cuartiles generales}

\begin{tabular}{|c|c|c|c|c|c|c|c|c|c|c|}
\hline & \multicolumn{5}{|c|}{ Mujeres } & \multicolumn{5}{|c|}{ Hombres } \\
\hline & \multicolumn{2}{|c|}{ Normal $(n=95)$} & \multicolumn{2}{|c|}{$C L(n=87)$} & \multirow[b]{2}{*}{$\mathbf{p}$} & \multicolumn{2}{|c|}{ Normal $(n=55)$} & \multicolumn{2}{|c|}{$C L(n=21)$} & \multirow[b]{2}{*}{ p* } \\
\hline & n & $\%$ & $\mathbf{n}$ & $\%$ & & $\mathbf{n}$ & $\%$ & $\mathbf{n}$ & $\%$ & \\
\hline$<32$ & 32 & 33,7 & 22 & 25,3 & 0,04 & 6 & 10,9 & 2 & 9,5 & 0,87 \\
\hline $33-48$ & 25 & 26,3 & 22 & 25,3 & & 13 & 23,6 & 4 & 19,0 & \\
\hline $49-61$ & 27 & 28,4 & 18 & 20,7 & & 12 & 21,8 & 6 & 28,6 & \\
\hline$>62$ & 11 & 11,6 & 25 & 28,7 & & 24 & 43,6 & 9 & 42,9 & \\
\hline
\end{tabular}

b. Distribución dicotómica de la edad (menores y mayores de 49)

\begin{tabular}{|c|c|c|c|c|c|c|c|c|c|c|}
\hline & \multicolumn{5}{|c|}{ Mujeres } & \multicolumn{5}{|c|}{ Hombres } \\
\hline & \multicolumn{2}{|c|}{ Normal $(n=95)$} & \multicolumn{2}{|c|}{$C L(n=87)$} & \multirow[b]{2}{*}{$\mathbf{p}$} & \multicolumn{2}{|c|}{ Normal $(n=55)$} & \multicolumn{2}{|c|}{$C L(n=21)$} & \multirow[b]{2}{*}{$\mathbf{p}^{\S}$} \\
\hline & n & $\%$ & $n$ & $\%$ & & n & $\%$ & $\mathbf{n}$ & $\%$ & \\
\hline$<49$ & 57 & 60,0 & 44 & 50,6 & 0,25 & 19 & 34,5 & 6 & 28,6 & 0,82 \\
\hline$>=49$ & 38 & 40,0 & 43 & 49,4 & & 36 & 65,5 & 15 & 71,4 & \\
\hline
\end{tabular}

CL: Colecistolitiasis. *Prueba de Jonckheere-Terpstra (basado en 10.000 réplicas). §Prueba de Chi cuadrado de Pearson. 
Tabla 3. Índice de masa corporal y hallazgos ecográficos en sujetos normales y con colecistolitiasis

\begin{tabular}{|c|c|c|c|c|c|c|}
\hline & & No & & & & \\
\hline & & $\mathbf{n}$ & $\%$ & $\mathbf{n}$ & $\%$ & $\mathbf{p}^{\S}$ \\
\hline IMC & $<30$ & 117 & 78 & 65 & 60,2 & $p<0,003$ \\
\hline & $>30$ & 33 & 22 & 43 & 39,8 & \\
\hline
\end{tabular}

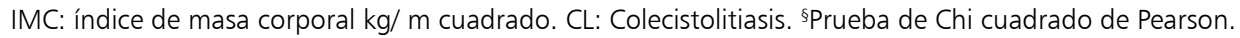

\section{Histología}

El análisis histopatológico de las vesículas extirpadas por colecistolitiasis de 106 pacientes sometidos a colecistectomia demostró que el hallazgo más frecuente fue la colecistitis crónica, seguido de la colesterolosis (habiendo en muchos casos más de un hallazgo histológico). No se encontró carcinoma en ningún paciente (Tabla 4).

\section{Discusión}

La formación de cálculos en la vesícula biliar tiene una etiología multifactorial que incluye factores genéticos y del medio ambiente. Estudios epidemiológicos muestran grandes diferencias de prevalencia entre diferentes poblaciones, donde destacan por su alta prevalencia los indios de América, notándose una disminución de la misma al ocurrir una mezcla con inmigrantes europeos $^{11,17-27}$. Se plantea para este hecho la presencia de genes litogénicos en la población indígena americana, los cuales son transmitidos a la población mestiza con ancestro amerindio ${ }^{28-31}$.

La población indígena de Chile se estima en 1,8 millones de personas, lo que equivale a $11 \%$ de la población total y se distribuye en 9 pueblos indígenas reconocidos. El pueblo mapuche tiene la mayor presencia con 1,18 millones $(86,4 \%)$, seguido por los aymaras con 99.270 personas $(7,2 \%)^{32}$. Ambas poblaciones comparten un alto índice de pobreza, bajo acceso a la atención hospitalaria y, específicamente, a la cirugía de colecistectomía ${ }^{33,34}$, factores que, junto con la presencia de colecistolitiasis y la obesidad, son los principales factores de riesgo para el desarrollo de cáncer de la vesícula biliar en Chile, con un O.R. de 5,1; 14,2; 3,9; y 7, respectivamente $e^{35,36,37}$. Aparentemente, por el alto número de población mapuche, hay una gran cantidad de información sobre esta etnia, pero ninguna referencia a la etnia aymara ${ }^{38}$.
Tabla 4. Informe histopatológico de 106 pacientes aymara con colecistolitiasis sometidos a colecistectomía

\begin{tabular}{|lcc|}
\hline Parámetro & n & \% \\
\hline Litiasis vesicular & 106 & 100 \\
Colecistitis crónica & 104 & 98 \\
\hline Colesterolosis & 32 & 30 \\
\hline Adenomiosis & 8 & 7,5 \\
\hline Cáncer de vesícula biliar & 0 & 0 \\
\hline
\end{tabular}

El presente estudio sugiere que hay una alta prevalencia de colecistolitiasis en la población aymara $(41,9 \%)$, que la posiciona como el pueblo originario con mayor índice de patología biliar benigna, por sobre la etnia mapuche, quienes presentan una prevalencia global de 35,2\% (Tabla 5). Estas altas cifras apoyan la hipótesis de la existencia de genes litogénicos en la población amerindia. Por otra parte, la asociación entre colecistolitiasis y CVB ha sido ampliamente documentada, la que en Chile es cercana a 100\%. Hallazgo similar se ha observado en otros pueblos indígenas de Norteamérica. Sin embargo, un estudio reciente sugiere que el riesgo de aparición de CVB no está asociado a cualquier tipo de ascendencia americana ${ }^{39,40}$. Lorenzo y cols., investigando una asociación entre esta ascendencia (basado en genotipos de todo el genoma) y las causas de muerte en los chilenos, observó que las dos etnias más numerosas en Chile (mapuches y aymaras), presentaban una relación inversa en el riesgo de desarrollar un CVB: mientras en los mapuches un aumento de $1 \%$ en la proporción de esta ascendencia se tradujo en un aumento de $3,7 \%$ en riesgo de mortalidad por CVB ( $\mathrm{p}=610-27)$, un aumento de $1 \%$ de ascendencia aymara se asoció a una disminución de $2,7 \%$ en el riesgo de $\mathrm{CVB}^{41}$. 
Tabla 5. Características generales y prevalencia global de cálculos biliares en dos pueblos indígenas de Chile

\begin{tabular}{|c|c|c|c|}
\hline & Mapuche $^{f}$ & Aymara & \\
\hline \multicolumn{4}{|l|}{ Género (n) } \\
\hline Mujeres & 92 & 182 & \\
\hline Hombres & 90 & 76 & \\
\hline Total & 182 & 258 & \\
\hline \multicolumn{4}{|l|}{ Edad (años) } \\
\hline Mujeres & $43 \pm 1,6$ & $46 \pm 16,4$ & \\
\hline Hombres & $47 \pm 1,8$ & $55 \pm 15,9$ & \\
\hline Total & $45 \pm 1,1$ & $48 \pm 16,8$ & \\
\hline \multicolumn{4}{|l|}{ IMC ( $\left.\mathrm{kg} \mathrm{m}^{-2}\right)$} \\
\hline Mujeres & $29 \pm 0,5$ & $28,2 \pm 4,7$ & \\
\hline Hombres & $26,3 \pm 0,3$ & $26,3 \pm 4,1$ & \\
\hline Colecistolitiasis (\%) ${ }^{\dagger}$ & & & $\mathbf{p}^{\S}$ \\
\hline Mujeres & 49,4 & 46,8 & 0,03 \\
\hline Hombres & 12,6 & 28,0 & \\
\hline Total & 35,2 & 41,2 & \\
\hline
\end{tabular}

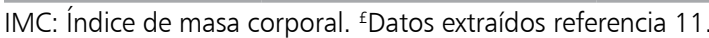
tPrevalencia ajustada por la edad en aymaras. §Prueba de Chi cuadrado de Pearson.

¿Por qué esta diferencia entre las dos etnias?. Datos epidemiológicos han sugerido que en las regiones donde se concentra la etnia aymara en Chile $(75,6 \%)$ hay una tasa de mortalidad por cáncer de vesícula biliar más baja que en el resto país (10,3 por cada 100.000 habitantes), a pesar de la alta prevalencia de colecistolitiasis, como se demuestra en el presente estudio. Por el contrario, la población aymara tiene $50 \%$ de mayor mortalidad por otros cánceres, que son la principal causa de muerte entre los aymaras ${ }^{14,42}$. En nuestra investigación no encontramos ningún CVB en 106 estudios histológicos, sin embargo, se podría argüir que la muestra estudiada es baja, pero en estudios de otras poblaciones con menor incidencia de colecistolitiasis, el hallazgo incidental de CVB en colecistectomías electivas es al menos de 3 a $5 \%{ }^{7,43,44}$.

Estos hallazgos histológicos que contrastan a los del pueblo mapuche, hacen surgir varias interrogantes: a) ¿estas diferencias se deben a los componentes genéticos, ambientales o ambos?; b) ¿hay algún factor de protección genético o ambiental específico para el desarrollo de CVB en esta etnia?; c) ¿qué mecanismos de prevención primaria y se- cundaria debieran establecerse en esta población para disminuir la tasa de colecistolitiasis?.

No es el propósito del presente estudio comentar la gran cantidad de genes litogénicos que se han descrito en la población nativa americana, ya que no fue nuestro objetivo, pero se han propuesto al menos 15 genes litogénicos en humanos, de los cuales el más estudiado es el gen de la apolipoproteina E (APOE) ${ }^{45-47}$. Recientemente, Bustos y cols. reportaron que los genes $A B C G 8$ y TRAF3 están asociados con ambos riesgos (cálculos vesiculares y CVB) en chilenos con ascendencia mapuche ${ }^{48}$. Sin embargo, debido a la importante asociación entre ambas patologías, se genera una gran dificultad al tratar de identificar factores de riesgo exclusivos para el CVB.

Respecto a los factores ambientales, se ha señalado que la población aymara vive en condiciones geográficas y con dietas diferentes, donde, en Colchane, el alimento principal es la llama (Lama glama), mientras que Camiña es un valle con mayor consumo de hortalizas. Sin embargo, no se demostró diferencias significativas en las dos poblaciones ${ }^{49-52}$. Datos epidemiológicos y experimentales sugieren que la exposición crónica a una gran altitud reduce la mortalidad por cáncer y la prevalencia de ciertos trastornos metabólicos como diabetes mellitus y obesidad, lo que implica un resultado aparentemente beneficioso para poblaciones asentadas en regiones geográficamente elevadas $^{53,54}$. Se postula que la mayoría de los procesos fisiológicos de adaptación a gran altitud ocurren en respuesta a la hipoxia, mediante la inducción o represión de la transcripción de diversos genes implicados en angiogénesis, metabolismo de la glucosa, metástasis, supervivencia o muerte celular ${ }^{55,56}$.

La prevención primaria de la colecistolitiasis se refiere al control de la obesidad e hipercolesterolemia en la poblacion aymara, mediante dieta baja en colesterol y alto en omega 3, y al control del síndrome metabólico, ya que por cada 5 puntos de aumento en el IMC, el riesgo de cálculos biliares aumenta 1,6 veces en mujeres. Esto es muy difícil de llevar a cabo, pero el realizar ecografías abdominales en la población de alto riesgo para desarrollar colecistolitiasis permitiría hacer un diagnóstico más precoz. Esta población de alto riesgo incluye a mujeres sobre 40 años, con IMC mayor a 30 y con comorbilidades como la diabetes mellitus. 
En conclusión, los resultados del presente estudio muestran una alta incidencia de colecistolitiasis en la población aymara, que es una etnia muy vulnerable, con altos niveles de pobreza, dificultad en el acceso al diagnóstico precoz de diferentes patologías y a la atención terciaria de salud. Todos estos factores, que en otras etnias indígenas producen un alto riesgo para el desarrollo de CVB, en los aymara no se ha documentado la misma alta incidencia de este carcinoma, sino por el contrario, su prevalencia parece ser muy baja. Se requerirá de una caracterización genética y ambiental más profunda (que incluya los efectos de la hipoxia) y comparativa entre los mapuches y aymaras que permita comprender mejor la patogénesis del CVB.

Agradecimientos: A Yolanda Flores y Jessica García del Departamento de Salud de la Comuna de Colchane y a todos los participantes del proyecto médico social de ayuda a la población aymara.

\section{Referencias}

1. Stinton L, Shaffer E. Epidemiology of gallbladder disease: cholelithiasis and cancer. Gut Liver 2012; 6,2: 172-87.

2. Cafasso D, Smith R. Symptomatic cholelithiasis and functional disorders of the biliary tract. Surgical Clinics 2014; 94.2: 233-256.

3. Andia M, Hsing A, Andreotti G, Ferreccio C. Geographic variation of gallbladder cancer mortality and risk factors in Chile: a population-based ecologic study. Int J Cancer 2008; 123.6: 1411-6.

4. Csendes A, Wunkhaus R, Santa Cruz V, Medina E. Frecuencia de litiasis biliar en autopsias. Arch Soc Ciruj Chile 1971; 23: 36-40.

5. Marinovic I, Guerra C, Larach G. Incidencia de litiasis biliar en material de autopsias y análisis de composición de los cálculos. Rev Med Chile 1972; 100: 1320-7.

6. Csendes A, Becerra M, Smok G, Medina E, Maluenda F, Morales E. Prevalencia del Cáncer de la vesícula biliar en colecistectomía. Rev Med Chile 1991; 119: 887-90.

7. Csendes A, Becerra M, Morales E. Carcinoma de la vesícula biliar. Trib Med 1991; 84: 131-6.

8. Csendes A, Cuneo N, Figueroa M, Orellana O. Evolución de operaciones por enfermedades del intestino proximal y glándulas anexas entre 2011 y 2016. Rev Chil Cir 2019; 71: 111-7.

9. Are C, Ahmad H, Ravipati A, Croo D, Clarey D, Smith $\mathrm{L}$, et al. Global epidemiological trends and variations in the burden of gallbladder cancer. J Surg Oncol 2017; 115 (5): 580-0.

10. Roa I, de Aretxabala X. Gallbladder cancer in Chile: what have we learned? Curr Opin Gastroenterol 2015; 31(3): 269-75.

11. Miquel JF, Covarrubias C, Villaroel L, Mingrone G, Greco A, Puglielli L, et al. Genetic epidemiology of cholesterol cholelithiasis amongst Chilean Hispanic, Amerindians, and Maoris. Gastroenterology 1998; 115: 937e946.

12. Vicuña MEG. Culturas indígenas de Chile: un estudio preliminar. Pehuén Editores Limitada. Santiago, Chile. 1998.

13. Van Kessel J. La cosmovisión aymara. En Hidalgo, H. L. Culturas de Chile Etnografía: Sociedades indígenas contemporáneas y su ideología, Santiago, Andrés Bello. 1989; 169-87.

14. Instituto Nacional de Estadísticas. Programa Orígenes. Estadísticas Sociales de los pueblos indígenas en Chile Censo 2002. 2005; Santiago de Chile.

15. Ministerio de Desarrollo Social, Gobierno de Chile (2009). Encuesta CASEN 2009.

16. Biblioteca del Congreso Nacional de Chile. Reportes Estadísticos Comunales (Colchane y Camiña). 2017 [cited $201925 \mathrm{Feb}$; Available from: https://reportescomunales.bcn.cl/2017/index.php/Página_principal.

17. Shaffer E. Epidemiology of gallbladder stone disease. Best Pract Res Clin Gastroenterol 2006; 20.6: 981-96.

18. Ratner J, Lisbona A, Rosenbloom M, Palayew M, Szabolcsi S, Tupaz T. The Prevalence of Gallstone Disease in Very Old Institutionalized Persons. JAMA 1991; 265 (7): 902-3.

19. Everhart J, Yeh F, Lee E, Hill M, Fabsitz R, Howard B, et al. Prevalence of gallbladder disease in American Indian populations: findings from the Strong Heart Study. Hepatology 2002; 35 (6): 1507-12.

20. Covarrubias C, Valdivieso V, Nervi F. Epidemiology of gallstone disease in Chile. In Capocaccia L, Ricci G, Angelico F, (eds.). Epidemiology and prevention of gallstone disease. Lancaster, England: MTP; 1984, pp. 26-30.

21. Moro PL, Checkley W, Gilman RH, Cabrera L, Lescano AG, Bonilla JJ, et al. Gallstone disease in Peruvian coastal natives and highland migrants. Gut 2000; 46 (4): 569-73.

22. Muhrbeck O, Ahlberg J. Prevalence of gallstone disease in a Swedish population. Scand J Gastroenterol 1995; 30: 1125-8.

23. Lirussi F, Nassuato G, Passera D, Toso S, Zalunardo B, Monica F, et al. Gallstone disease in an elderly population: the Silea study. Eur J Gastroenterol Hepatol 1999; 11(5): 485-91. 
24. Khuroo MS, Mahajan R, Zargar S A, Javid G, Sapru S. Prevalence of biliary tract disease in India: a sonographic study in adult population in Kashmir. Gut 1989; 30 (2): 201-5.

25. Lu SN, Chang WY, Wang LY, Hsieh MY, Chuang WL, Chen SC, et al. Risk factors for gallstones among Chinese in Taiwan. A community sonographic survey. J Clin Gastroenterol 1990; 12: 542-6.

26. Zhao Y, Zhang R, Hu Y, Li R, Liang L, Gang Y. An epidemiological survey of gallstones with gray-scale ultrasound. Hua Xi Yi Ke Da Xue Xue Bao (J West China Univer Med Sci) 1990; 21: 217-20.

27. Bagi Abdel M, Arabi M, Abdel Rahim B. Prevalence of gallbladder disease in Sudan: first sonographic field study in adult population. Gastroenterology 1991; 100: A307.

28. Everhart J, Yeh F, Lee E, Hill M, Fabsitz R, Howard V, et al. Prevalence of gallbladder disease in American Indian populations: findings from the Strong Heart Study. Hepatology 2002; 35(6): 1507-12.

29. Francisco J, Poblete M. Salud y enfermedad en nuestras poblaciones originarias: qué los hace ser más susceptibles o resistentes a ciertas enfermedades prevalentes. Rev Chil Cardiol 2012; 31 (2): 129-33.

30. Nervi F, Miquel J, Marshall G. The Amerindian epidemics of cholesterol gallstones: the North and South connection. Hepatology 2003; 37 (4): 947-8.

31. Grundy S, Metzger A, Adler, R. 1972 Mechanisms of lithogenic bile formation in American Indian women with cholesterol gallstones. J Clin Invest 1972; 51 (12): 3026-43.

32. Instituto Nacional de Estadísticas. Radiografía de genero: Pueblos originarios en Chile 2017. Unidad de estudios y estadísticas de género, Instituto Nacional de estadísticas 2018.

33. Ministerio de Desarrollo Social, Gobierno de Chile (2018). Encuesta CASEN 2017.

34. Del Popolo F, Dirk J, NU CEPAL, Los pueblos indígenas en América Latina: Avances en el último decenio y retos pendientes para la garantía de sus derechos. 2014. Santiago de Chile: Impreso en Naciones Unidas.

35. Andia M, Hsing W, Andreotti G, Ferreccio C. Geographic variation of gallbladder cancer mortality and risk factors in Chile: a population-based ecologic study. Int J Cancer 2008; 123 (6): 1411-6.

36. Villanueva L. Cancer of the gallbladder-Chilean statistics. Ecancermedicalscience 2016; 10: 704.

37. Roa I, Araya J C, Villaseca M, Roa J, Ibacache G. Gallbladder cancer in a high risk area: morphological features and spread patterns. Hepatogastroenterology 1999; 46 (27): 1540-6.
38. Andia M, Gederlini A, Ferreccio C. Cáncer de vesícula biliar: Tendencia y distribución del riesgo en Chile. Rev Med Chile 2006; 134: 565-74.

39. Shukla V, Khandelwal C, Roy S, Vaidya M. Primary carcinoma of the gall bladder: A review of a 16-year period at the university hospital. J Surg Oncol 1985; 28 (1): 32-5.

40. Nemunaitis J, Brown-Glabeman U, Soares H, Belmonte J, Liem B, Nir I, et al. Gallbladder cancer: review of a rare orphan gastrointestinal cancer with a focus on populations of New Mexico. BMC Cancer 2018; 18 (1): 665.

41. Lorenzo J, Boekstegers F, González R, Marcelain K, Baez P, et al. Subtypes of Native American ancestry and leading causes of death: Mapuche ancestry-specific associations with gallbladder cancer risk in Chile. PLoS genetics 2017; 13(5): e1006756.

42. SEREMI de Salud Arica y Parinacota, Situación de salud de la población Aymara en la región de Arica y Parinacota. Evidencias de inequidades étnicas en el norte de Chile. First ed. 2014, Arica.

43. Silecchia G, Raparelli L, Navalone J, Serantes A, Moreno M, Materia A, et al. Laparoscopic cholecystectomy and incidental carcinoma of the extrahepatic biliary tree. JSLS 2002; 6: 339-44.

44. Castillo J, Csendes A, Díaz J C, Sauré A, Guajardo M, Lembach H. Cáncer temprano de la vesícula biliar: tratamiento y sobrevida a largo plazo. Rev Chil Cir 2014; 66: 417-22.

45. Krawczyk M, Miquel J F, Stokes C, Zuniga S, Hampe J, Mittal B, et al. Genetics of biliary lithiasis from an ethnic perspective. Clin Res Hepatol Gastroenterol 2013; 37 (2): 119-25.

46. Weber S, Bopp C, Krawczyk M, Lammert F. Genetics of gallstone disease revisited: updated inventory of human lithogenic genes. Curr Opin Gastroenterol 2019; 35 (2): 82-7.

47. Wang H, Li T, Portincasa P, Ford D, Neuschwander-Tetri B, Tso P, et al. New insights into the role of Lith genes in the formation of cholesterol-supersaturated bile. Liver Research 2017; 1 (1): 42-53.

48. Bustos B, Pérez-Palma E, Buch S, Azócar L, Riveras E, Ugarte $\mathrm{G}$, et al. Variants in ABCG8 and TRAF3 genes confer risk for gallstone disease in admixed Latinos with Mapuche Native American ancestry. Sci Rep 2019; 9 (1): 772 .

49. Negri E, La Vecchia C, Franceschi S, D’Avanzo B, Parazzini $\mathrm{F}$. Vegetable and fruit consumption and cancer risk. Int J Cancer 1991; 48 (3): 350-4.

50. Serra I, Yamamoto M, Calvo A, Cavada G, Baez S, Endoh $\mathrm{K}$, et al. Association of chili pepper consumption, 
low socioeconomic status and longstanding gallstones with gallbladder cancer in a Chilean population. Int J Cancer 2002; 102 (4): 407-11.

51. Pandey M, Shukla VK. Diet and gallbladder cancer: a case-control study. Eur J Cancer Prev 2002; 11 (4): 365-8.

52. Navarro D, Durán S. Cáncer de vesícula biliar en Chile y factores nutricionales de riesgo. Nutr Hosp 2016; 33.1: 105-10.

53. Thiersch M, Swenson E R, Haider T, Gassmann, M. Reduced cancer mortality at high altitude: The role of glucose, lipids, iron and physical activity. Exp Cell Res 2017; 356.2: 209-16.

54. Boticario C, Angosto M. Hipoxia y cáncer. An de la Real Acad Nac de Farm 2010; 76(3).

55. Kenneth N, Rocha S. Regulation of gene expression by hypoxia. Biochem J (2008) 414 (1): 19-29.

56. García-Heredia J M, Felipe-Abrio B, Cano DA, Carnero A. Genetic modification of hypoxia signaling in animal models and its effect on cancer. Clin transl oncol 2015; 17: 90-102. 